\title{
Modelling Longitudinal Metabonomics and Microbiota Interactions in C57BL/6 mice fed a high fat diet
}

Running title: Longitudinal integration of Metabonomics and Microbiota

Ivan Montoliu, ${ }^{1,2 \#}$ Ornella Cominetti, ${ }^{1 \#}$ Claire L. Boulangé, ${ }^{2}$ Bernard Berger, ${ }^{3}$ Jay Siddharth, ${ }^{1}$ Jeremy Nicholson, ${ }^{2}$ François-Pierre J. Martin ${ }^{1}$

${ }^{\mathbf{1}}$ Nestlé Institute of Health Sciences SA, EPFL Innovation Park, Building H,1015 Lausanne, Switzerland; ${ }^{2}$ Department of Biomolecular Medicine, Division of Surgery, Oncology, Reproductive Biology and Anaesthetics, Faculty of Medicine, Imperial College London, Sir Alexander Fleming Building, South Kensington Campus, London SW7 2AZ, UK; ${ }^{3}$ Nestlé Research Center, Vers-chez-les-Blanc, 1000 Lausanne 26, Switzerland;

\section{Supporting Information}

Supporting Information Available: 5 Supplementary Figures and 2 Supplementary Tables. 
Table S1: Summary of model parameters for group comparison

\begin{tabular}{|l|l|l|l|l|}
\hline $\begin{array}{l}\text { Model / Diet } \\
\text { comparison }\end{array}$ & Factors & $\mathbf{R}^{2} \mathbf{X}$ & $\mathbf{R}^{2} \mathbf{Y}$ & $\mathbf{Q}^{2} \mathbf{Y}$ \\
\hline LF $\mid$ HF & $1+2+0$ & 0.529 & 0.699 & 0.644 \\
\hline LF $\mid$ HF_S & $1+3+0$ & 0.566 & 0.568 & 0.473 \\
\hline HF $\mid$ HF_S & $1+1+0$ & 0.442 & 0.685 & 0.645 \\
\hline HF $\mid$ HF + GOS & $1+2+0$ & 0.533 & 0.743 & 0.699 \\
\hline HF $\mid$ HF + BMOS & $1+2+0$ & 0.557 & 0.736 & 0.689 \\
\hline HF $\mid$ HF + FOS_IN & $1+1+0$ & 0.514 & 0.683 & 0.656 \\
\hline HF_S $\mid$ HF + BMOS & $1+2+0$ & 0.566 & 0.605 & 0.524 \\
\hline
\end{tabular}


Table S2: Summary of metabolites associated with body weight with the different feeding models

\begin{tabular}{|c|c|c|c|c|c|c|}
\hline Model / Metabolites & $\begin{array}{l}\mathrm{HF} \mid \\
\mathrm{HF} \mathrm{S}_{2}\end{array}$ & $\begin{array}{l}\mathrm{HF} \mid \\
\mathrm{HF}+\mathrm{GOS}\end{array}$ & $\begin{array}{l}\mathrm{HF} \mid \\
\mathrm{HF}+\mathrm{GOS}\end{array}$ & $\begin{array}{l}\mathrm{HF} \mid \\
\mathrm{HF}+\mathrm{FOS} \text { IN }\end{array}$ & $\begin{array}{l}\mathrm{HF} \mid \\
\mathrm{HF}+\mathrm{BMOS}\end{array}$ & $\begin{array}{l}\text { HF_S } \mid \\
\mathrm{HF}+\mathrm{BMOS}\end{array}$ \\
\hline $\begin{array}{l}\alpha \text {-keto- } \\
\text { methylvalerate }\end{array}$ & $\mathrm{De}$ & & & & & \\
\hline$\alpha$-ketoisovalerate & $\mathrm{De}$ & & & & & \\
\hline Acyl-carnitine & & In & In & & & \\
\hline Carnitine & In & In & In & In & De & De \\
\hline Creatine & & In & In & In & & \\
\hline Creatinine & In & In & In & In & In & In \\
\hline Guanidoacetate & In & & & In & & \\
\hline Hippurate & In & & & In & In & \\
\hline Indoxyl-sulfate & & In & In & In & In & In \\
\hline Isobutyrate & $\mathrm{De}$ & & & & & \\
\hline Leucine & $\mathrm{De}$ & & & & & \\
\hline Nicotinate & In & In & In & In & In & In \\
\hline Nicotinurate & In & In & In & In & In & In \\
\hline Oxaloacetate & & In & In & In & In & In \\
\hline Phenylacetylglycine & & In & In & In & In & In \\
\hline Sucrose & & In & In & & & De \\
\hline Taurine & & & & De & & \\
\hline Tartarate & In & In & In & In & In & In \\
\hline TMA & & & & & De & De \\
\hline TMAO & & & & & De & $\mathrm{De}$ \\
\hline
\end{tabular}

Key: In, Increase; De, Decrease; 
Figure S1: Body weight variations observed with the different nutritional intervention. Data are reported as body weight average at each weekly visit. Key: LF: low fat, HF: high fat, Dx: Visit time in days before or after diet switch (D0).

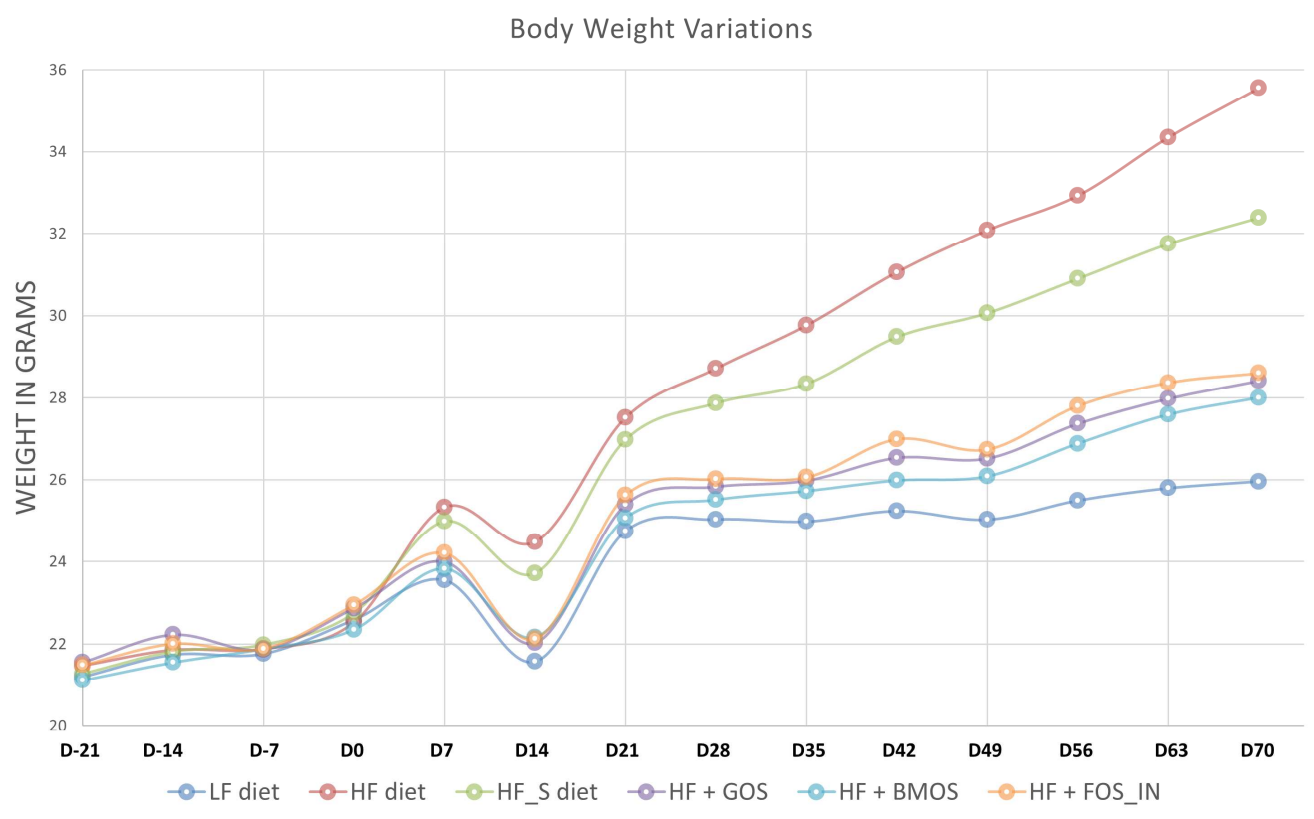


Figure S2: PLS Model comparison for HF $\mid \mathrm{HF} S \mathrm{~S}$ with $\mathrm{HF} \mid \mathrm{HF}+$ prebiotics diets. Metabolite associated variables on the diagonal had similar importance in explaining body weight variance in the two models. A threshold value of 0.2 was applied to select the most influential variables. This selection corresponds mostly to values of VIP for the variable on any of both models $>=1.0$.

Figure S1

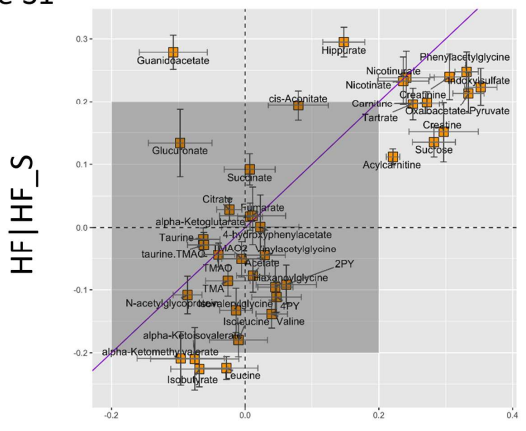

$\mathrm{HF} \mid \mathrm{HF}+\mathrm{GOS}$

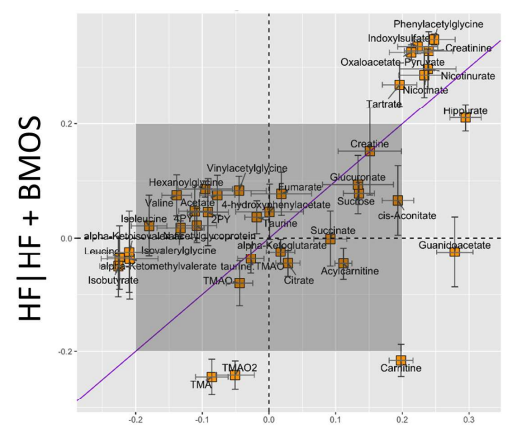

HF $\mid$ HF_S

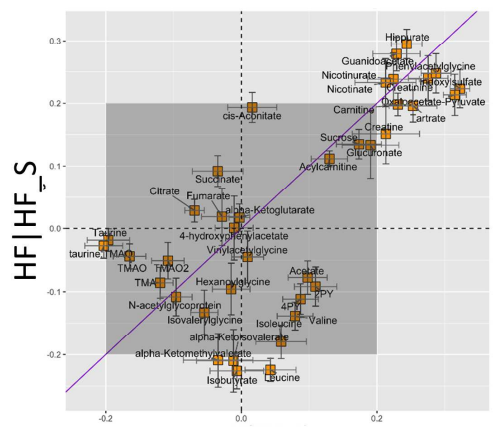

HF $\mid H F+F O S \_I n$ 
Figure S3: PLS Model comparison for $\mathrm{HF} \mid \mathrm{HF}+\mathrm{GOS}$ with $\mathrm{HF} \mid \mathrm{HF}+\mathrm{BMOS}$, and $\mathrm{HF} \mid \mathrm{HF}+\mathrm{GOS}$ with HF $\mid \mathrm{HF}+$ FOS_IN.

Metabolite associated variables on the diagonal had similar importance in explaining body weight variance in the two models. A threshold value of 0.2 was applied to select the most influential variables. This selection corresponds mostly to values of VIP for the variable on any of both models $>=1.0$.
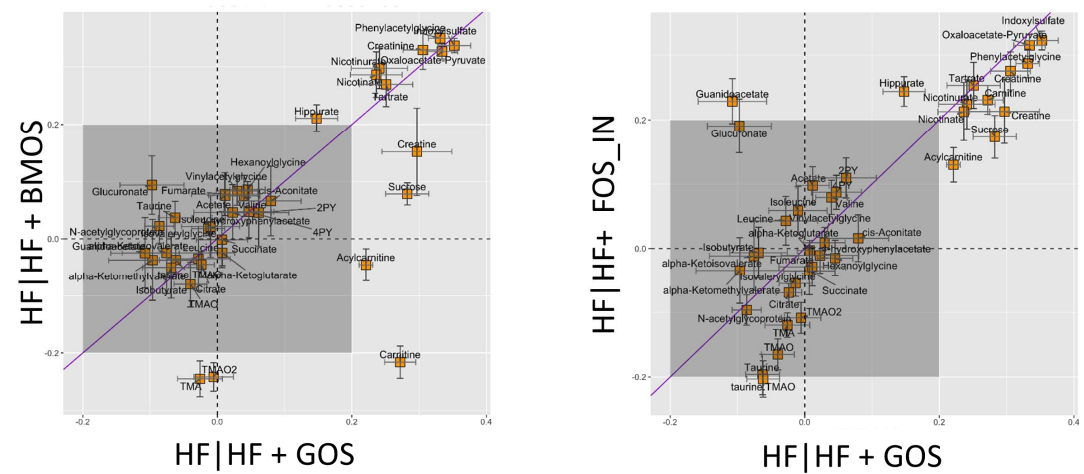
Figure S4: PLS Model comparison for HF $\mid \mathrm{HF}+$ BMOS with HF_S $\mid \mathrm{HF}+\mathrm{BMOS}$, and $\mathrm{HF} \mid \mathrm{HF}+$ BMOS with HF $\mid \mathrm{HF}+$ FOS_IN.

Metabolite associated variables on the diagonal had similar importance in explaining body weight variance in the two models. A threshold value of 0.2 was applied to select the most influential variables. This selection corresponds mostly to values of VIP for the variable on any of both models $>=1.0$.
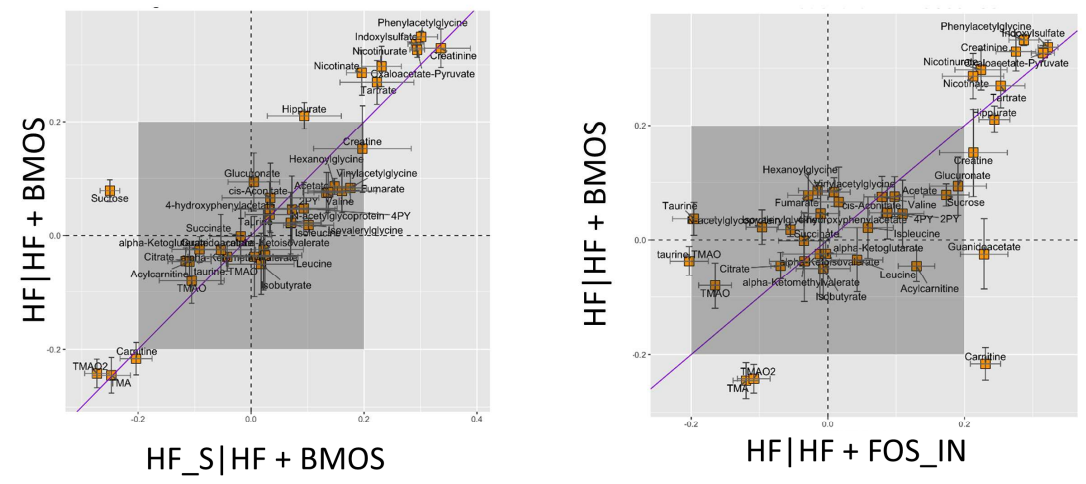
Figure S5: Curves of number of variables (A metabolites and B microbiota species) for increasing similarity score thresholds. No single pair of variables have high similarity score thresholds for at least one of the groups $(>0.7)$ and the lowest similarity score for all variables is 0.2 , below which all the variables are selected for at least one dietary group. The blue vertical lines depict the thresholds chosen which give a reasonable number of variables to visually inspect and compare via heatmaps.

A

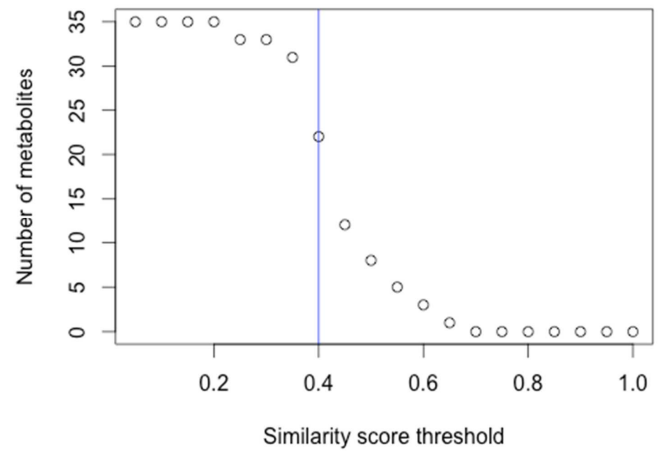

B

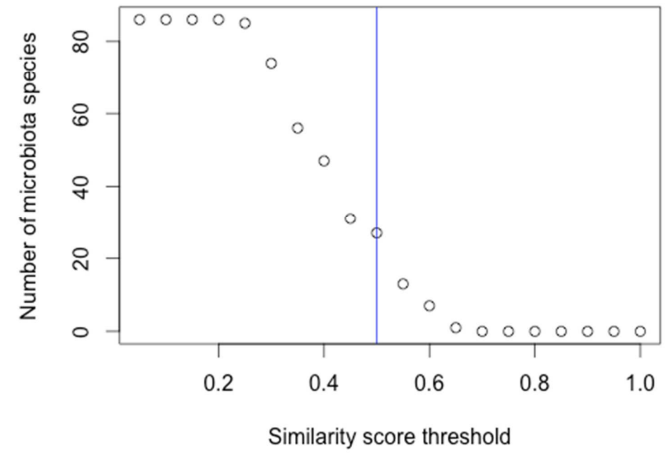

